\title{
Axiom-based Potential Functional Failure Analysis for Risk-free Design
}

\author{
Lian Benning, Cao Guozhong, Bai Zhonghang, and Tan Runhua \\ Hebei University of Technology, Department of Mechanical Engineering, \\ Guang Rong Road NO.8, PO Box: 165, 300130 Tianjin, P.R. China \\ Phone: +86-22-60204871, Fax: +86-22-26564037 \\ E-mai: lianbenning@126.com
}

\begin{abstract}
Quality and reliability of product is not established completely in detail design process, but is brought out essentially in the course of conceptual design. A potential functional failure analysis method to improve reliability of product based on design axiom in the stage of conceptual design was introduced in this paper. This method provided designers with an analytical and non-probabilistic tool to evaluate the result of conceptual design from the opinion of design axioms. Functional failure modes can be identified by function analysis based on the ideality axiom, the independence axiom and the information axiom. These potential functional failures point out working direction for the latter improving design. A speedy cutting off valve in the TR' (Top Gas Pressure Recovery Turbine) system is studied as an example to illustrate this method's potential.
\end{abstract}

\section{Introduction}

In product design, the end goal is to develop a product which performs a function or functions to satisfy the customer's needs. Pahl and Beitz [1] state that nearly $\mathbf{8 0 \%}$ of the costs and problems are created in product development and that cost and quality are essentially designed into products in the conceptual stage. As to produce a more reliable product without the need for multiple redesigns, it is so significant to begin the failure analysis of a product design to prevent failure modes in advanced stage of the design process. Classical failure analysis techniques such as Failure Modes and Effects Analysis (FMEA), Anticipatory Failure Determination (AFD) and Fault Tree Analysis (FTA) are used in currently industries to determine potential failures of products [2-4]. In order to eliminate or reduce the possibility of failure, designers need to be aware of all of the potential significant failure modes in the systems being designed. An essential and crucial part of these methods is a required function-failure knowledge base of previous products. Tumer and Stone [5] collected historical failure data to establish a connection between failure modes and product's

Please use the following format when citing this chapter.

Benning, L., Gouzhong, C., Zhonghang, B., Runhua, T., 2007, in IFIP International Federation for Information Processing, Volume 250, Trends in Computer Aided Innovation, ed. León-Rovira, N., (Boston: Springer), pp. 107-114. 
functions. Also some other researchers did a considerable amount of work in developing the function-failure knowledge base in their respective field [6-8]. In this paper, an axiom-based functional failure analysis method for risk-free design is presented in functional design of product to change the experience-based functional failure analysis into the use of scientific theories and methodologies that based on design axioms.

There are two ways to deal with design: experiment-based and axiom-based. In essence, classical failure analysis techniques mentioned above are experiment-based approaches because they needed a mass of failure data which should be collected in real application or experiments. The experiment-based approach is generally useful at the detail level. However, it is difficult to match failure data to product functions very well, for there is no detail structure information of product in the conceptual design, especially in functional design. The axiom-based approach to design is based on the abstraction of good design decisions which is easy to be reused in the conceptual design process.

\section{Design Axioms}

Axioms are established rules, principles, or laws. Fields such as mathematics, physical science, and engineering have gone through the transition from experiencebased practice to the use of scientific theories and methodologies that are based on axioms. Altshuller has introduced the ideality axiom in the theory of inventive problem solving (TRIZ) [9]. Suh has presented two design axioms in his axiomatic design approach [10]. The first design axiom is known as the independence axiom and the second axiom is known as the information axiom. All these design axioms are stated as follows:

- Axiom 1: The Ideality Axiom

Since technological system are designed to perform certain function or functions, a better system obviously requires less material to built and maintain, and less energy to operate, to perform these functions. Altshuller pointed out that an ideal technological system is a system whose mass, dimensions, cost, energy consumption, etc. are approaching zero, but whose capability to perform the specified function is not diminishing [9]. The ideality axiom states that the design with the highest ideality is the best design. The ideality of a product or a technical system is one of the major criterions when we assess the quality of design.

- Axiom 2: The Independence Axiom

In axiomatic design, the design objective is defined in terms of functional requirements (FRs) and the physical solution is characterized in terms of design parameters (DPs). Design task can be represented as a mapping between functional domain and physical domain as show in Fig. 1. The independence axiom states that mapping between the FRs and DPs in an acceptable design is such that each FR can be satisfied without affecting any other FRs.

- Axiom 3: The Information Axiom

The information axiom states that the design with the highest probability of achieving the design goals as expressed by the functional requirements (FRs) is the best design. The system performance is shown by its probability density function (pdf), which defines the system range. The FR is satisfied only when the system rang 
is within the design range. The overlap between design rang and system range is called common range, which is the only region where the FR is satisfied as shown in Fig. 2.

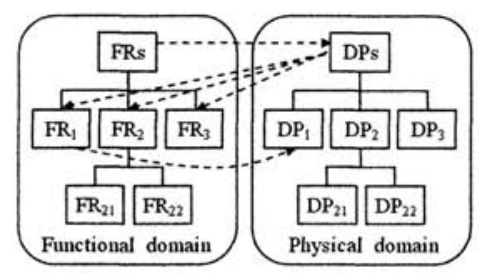

Fig. 1. Zigzagging to decompose FRs and DPs in the functional and physical domains to create the FRs and DPs hierarchies.

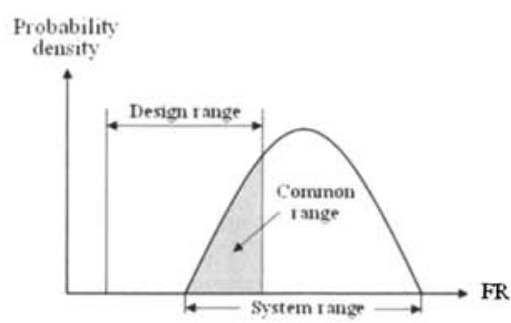

Fig. 2. Design range, system range, common range and pdf for an FR.

\section{Axiom-based Functional Failure}

\subsection{Functional Failure based on Axiom 1}

One principle of TRIZ, and a method of determining the progress is the ideality axiom [11]. Ideality is summarized by the equation:

$$
\text { Ideality }=\sum \mathrm{UF} / \sum \mathrm{HF}
$$

where $\sum$ UF is the sum of all useful functions and $\sum$ HF is the sum of all harmful functions. In fact, a technique is a "fee" for realization of the required function, because a technique always has harmful functions. For example, when we have our car braked by brakes, the noises and heat what we do not need will be produced. If the degree of the ideality is too low, that will cause the whole system to fail. 


\subsection{Functional Failure based on Axiom 2}

In axiomatic design, the mapping between functional domain and physical domain can be described mathematically by the following equation:

$\{\mathrm{FR}\}=|\mathrm{A}|\{\mathrm{DP}\}$

where $\{F R\}$ is the functional requirement vector, $\{D P\}$ is the design parameter vector, and $|\mathrm{A}|$ is the design matrix that characterizes the design. When the $|\mathrm{A}|$ matrix is a diagonal matrix, each DP can be decided independently by its respective FR. When the $|A|$ matrix is a triangular matrix, DP can be decided independently by following the order of determination. Otherwise, when the $|A|$ matrix has no special structure, one function failing to satisfy its DP will cause many other functions to be performed unsuccessfully.

\subsection{Functional Failure based on Axiom 3}

The information axiom indicates that the variance of the system must be small and the bias must be eliminated to make the system range lie inside the design range for enhancing robust of the design. The insufficient useful action or the excessive useful action of the FR will cause the respective function to fail when the bias between the system range and the design range overruns the limit.

\section{Case Study: a Speedy Cutting Off Valve}

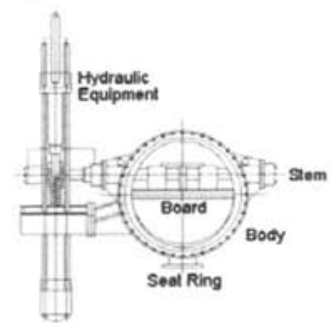

Fig. 3. Structure of the speedy cutting off valve.

In order to use excessive blast furnace gas, a kind of equipment called TRT (Top Gas Pressure Recovery Turbine) is developed. Speedy cutting off valve is used for urgent cutting off the TRT, as shown in Fig. 3. From the point of view of system function, the Fig. 4 shows the problems of the speedy cutting off valve in TRT. Three kinds of functional failure modes can be identified based on design axioms. The harmful actions: the dust polluting the oil, the filter and the pipeline counteracting the fluid of oil, the hydraulic cylinder counteracting the movement of the piston; The insufficient action: the dynamical spring driving the piston deficiently; The excessive actions: the motor and the hydraulic control box consuming massive electricity supply. 
All these functional failures mentioned above point out working direction for the latter improving design. The improved function model of the speedy cutting off valve is shown in Fig. 5. In the improved drive system the hydraulic devices are removed completely and a new drive device is adopted shown in Fig. 6 . The system can realize all motions, such as the slow opening, slow shutting, quick shutting and moving about movements, as well as the buffering and the complete closure of valve.

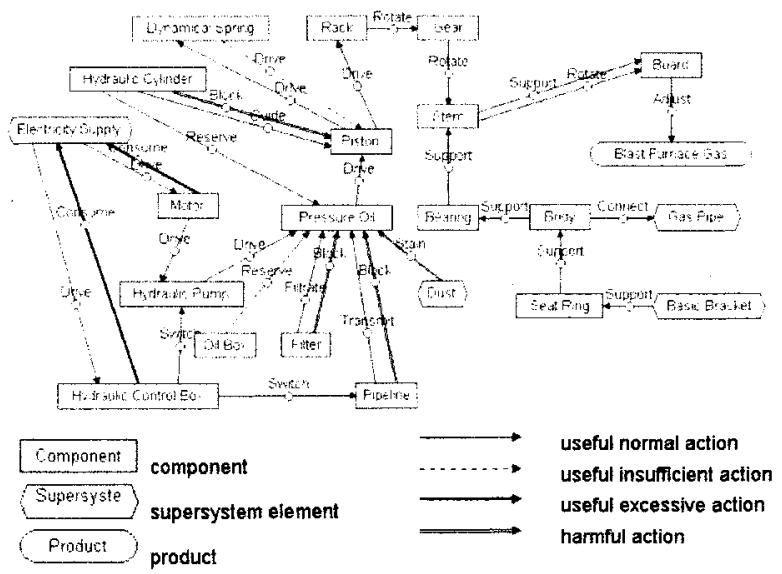

Fig. 4. Structure of the speedy cutting off valve.

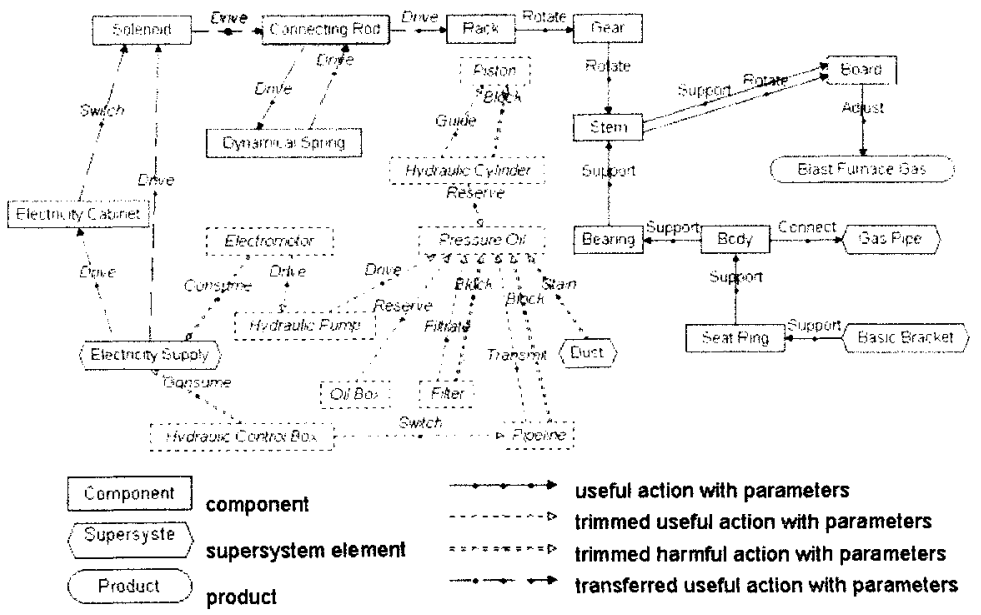

Fig. 5. Improved Function Model of the Speedy Cutting Off Valve 


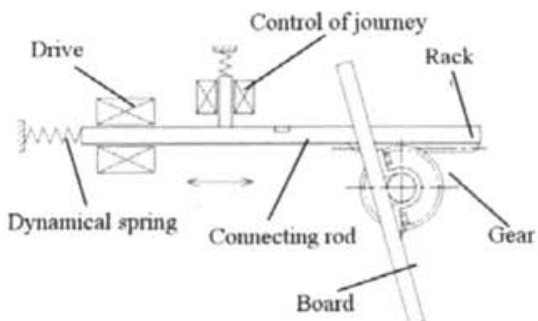

Fig. 6. Principle Solution of the New Drive System

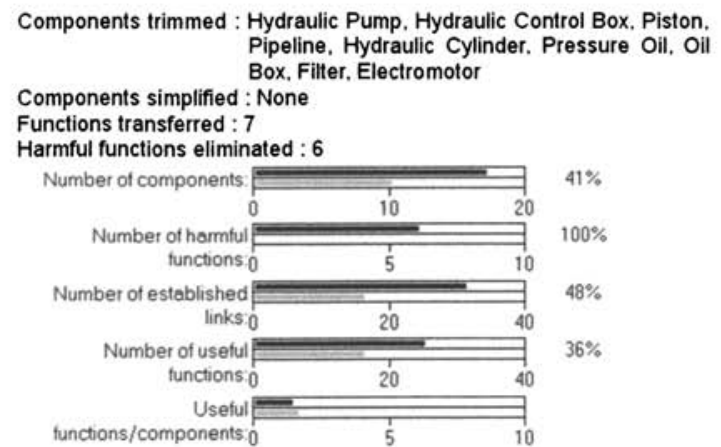

Fig. 7. Improvement Result of the Speedy Cutting Off Valve

The FRs and DPs of the new drive system as shown in Fig. 6 are:

$\mathrm{FR}_{1}$ : supply power $\mathrm{DP}_{1}$ : power device

$\mathrm{FR}_{2}$ : transfer power $\quad \mathrm{DP}_{2}$ : transfer device

$\mathrm{FR}_{3}$ : control power $\quad \mathrm{DP}_{3}$ : control device

The relation of FRs to DPs is:

$\left\{\begin{array}{l}\mathrm{FR}_{1} \\ \mathrm{FR}_{2} \\ \mathrm{FR}_{3}\end{array}\right\}=\left[\begin{array}{lll}1 & 0 & 0 \\ 1 & 1 & 0 \\ 1 & 1 & 1\end{array}\right]\left\{\begin{array}{l}\mathrm{DP}_{1} \\ \mathrm{DP}_{2} \\ \mathrm{DP}_{3}\end{array}\right\}$

The $\mathrm{FR}_{1}$ and $\mathrm{DP}_{1}$ can be more divided into:

$\mathrm{FR}_{11}$ : supply closing power $\quad \mathrm{DP}_{11}$ : closing device

$\mathrm{FR}_{12}$ : supply opening power $\mathrm{DP}_{12}$ : opening device

The relation of FRs to DPs is:

$\left\{\begin{array}{l}\mathrm{FR}_{11} \\ \mathrm{FR}_{12}\end{array}\right\}=\left[\begin{array}{ll}1 & 0 \\ 1 & 1\end{array}\right]\left\{\begin{array}{l}\mathrm{DP}_{11} \\ \mathrm{DP}_{12}\end{array}\right\}$

According to the two design equations, the requirements satisfy the Independence Axiom. There is no coupled functional failure in the new driving system of the improved speedy cutting off valve.

In this case, comparing the improved system with the original system as shown in Fig. $7,33 \%$ of elements and $43 \%$ of interactions between elements are decreased, 
potential functional failures are eliminated completely, the system structure is simplified and the cost is reduced. From the view of function, the negative effects aroused by the hydraulic system, such as the pollution of oil, the flow resistance of oil, the unreliability of hydraulic meter, deficient force of spring and etc., are removed in the original system by substituting the hydraulic drive system with the new electromagnetic drive system. Every movement of the speedy cutting off valve can be implemented well, the operation is much simpler and the system performance is improved greatly.

\section{Conclusion}

Using the idea of axiom, in a larger way than the idea of statistics, it is possible to apply the functional failure analysis in the conception design when there is lack of function-failure data collected by a great lot of failure reports or reliability experiments. In this paper, a functional failure analysis method based on design axioms was introduced to improve reliability and performance of products in the stage of conceptual design.

According to the ideality axiom, all the harmful function should be removed to increase the ideality of the system. The independence axiom indicates that the coupled design is undesirable, for the change in any DP may cause many FRs to fail simultaneously. The information axiom points out that the insufficient useful action or the excessive useful action of the FR will cause the respective function to fail when the bias between the system range and the design range overruns the limit. Therefore, we identify and then eliminate all these potential functional failures when functional model is constructed to improve the reliability and performance of products.

In the case study of the speedy cutting off valve in the TRT, comparing the improved system with the original system proves that this method is feasible.

\section{Acknowledgment}

This research is supported in part by the Natural Science Foundation of China under Grant Numbers 50675059 and the National High Technology Research and Development Program of China under Grant Numbers 2006AA042109. Any opinions or findings of this work are the responsibility of the authors, and do not necessarily reflect the views of the sponsors or collaborators.

\section{References}

1. G. Pahl and W. Beitz, Engineering Design: A Systematic Approach (Springer Verlag, 1996).

2. A. Carter, Mechanical Reliability and Design (Wiley, 1997).

3. E. Henley and H. Kumamoto, Probabilistic Risk Assessment: Reliability Engineering, Design and Analysis, IEEE (1992). 
4. I. Tumer and R. Stone, Analytical Method for Mapping Function to Failure during Highrisk Component Development, in: ASME Design for Manufacturing Conference, DETC2001/DFM-21173, Pittsburgh, PA (2001).

5. I.Y. Tumer and R.B. Stone, Mapping Function to Failure during High-risk Component Development, Research in Engineering Design, 14(1), 25-33 (2003).

6. S.G. Arunajadai, R.B. Stone, and I.Y. Tumer, A Framework for Creating a Function-based Design Tool for Failure Mode Identification, in: Proceedings of the 2002 ASME Design Engineering Technical Conference, Design Theory and Methodology Conference, Montreal, Canada (2002).

7. R.A. Roberts, R.B. Stone, and I.Y. Tumer, Deriving Function-Failure Information for Failure-free Rotorcraft Component Design, in: Proceedings of the 2002 ASME Design Engineering Technical Conferences, Design for Manufacturing Conference, DETC2002/DFM-34166, Montreal, Canada (2002).

8. I.Y. Tumer, R.B. Stone, R.A. Roberts, and A.F. Brown, A Function-based Exploration of JPL's Problem/Failure Reporting Database, Proceedings of the 2003 ASME International Mechanical Engineering Congress and Expo, IMECE2003-42769, Washington (2002).

9. V. Fey and E.I. Rivin, Innovation on Demand: New Product Development Using TRIZ (Cambridge University, 2005).

10. N.P. Suh, The Principles of Design (Oxford University, 1990).

11. S.D. Savransky, Engineering of Creativity: Introduction to TRIZ Methodology of Inventive Problem Solving (CRC, 2000). 